more variation in reports of minority taxa and relative abundances. Standardized use of mock communities may improve reproducibility across vaginal microbiota studies.

Disclosure No significant relationships.

\section{P589 THE INFLUENCE OF PRECONCEPTION VAGINAL MICROBIOTA ON PRETERM BIRTH}

'Kalpana Betha, ${ }^{2}$ Srinivas Vudathala, ${ }^{3}$ Saumyadipta Pyne, ${ }^{1}$ Govind Kusneniwar, ${ }^{1}$ Pavani Sowjanya, ${ }^{3}$ PS Reddy, ${ }^{4}$ Catherine Haggerty. ${ }^{1}$ SHARE India, Telangana, India ${ }^{2}$ PathCare Labs Pvt Ltd., Telangana, India; ${ }^{3}$ University of Pittsburgh, Pittsburgh, USA; ${ }^{4}$ University of Pittsburgh, Graduate School of Public Health, Department of Epidemiology, Pittsburgh, USA

10.1136/sextrans-2019-sti.660

Background Preterm birth (PTB) is common worldwide and causes significant neonatal morbidity. Although ascending reproductive tract infection has been implicated in approximately half of spontaneous PTB cases, the microbiologic etiology remains poorly understood and no studies have examined the role of preconception vaginal microbiota in PTB.

Methods We conducted a pilot study comparing bacterial communities among 6 women who experienced a PTB $<34$ weeks' gestation and 12 term delivery controls who participated in the Longitudinal Indian Family hEalth (LIFE) study in Telangana, India. Archived preconception vaginal samples were analyzed using broad-range $16 \mathrm{~S}$ rRNA gene PCR with sequencing. Women with preeclampsia were excluded.

Results Cases had more sequence reads from Sneathia spp., Megasphaera spp., and Atopobium vaginae than controls. Overall, the vaginal microbiota of cases was more diverse than those from controls. Women who delivered at term generally had vaginal microbiota dominated by Lactobacillus spp.

Conclusion Our study suggests key differences in preconception vaginal bacterial communities between women who experience a PTB compared to women who deliver at term. Future large scale epidemiologic studies of preconception and prenatal vaginal microbiota and adverse pregnancy outcomes are warranted and may guide PTB interventions.

Disclosure No significant relationships.

\section{P590 VAGINAL MICROBIOTA AND DOUCHING CESSATION: A CROSSOVER PILOT STUDY}

${ }^{1}$ Sarah Brown, ${ }^{2}$ Xin He, ${ }^{1}$ Courtney Robinson, ${ }^{3}$ Khalil Ghanem, ${ }^{1}$ Jacques Ravel, ${ }^{3} J o n a t h a n$ Zenilman, ${ }^{1}$ Rebecca Brotman. 'University of Maryland, Baltimore, Institute for Genome Sciences, Baltimore, USA; ${ }^{2}$ University of Maryland, College Park, Epidemiology and Biostatistics, College Park, USA; ${ }^{3}$ Johns Hopkins, Infectious Diseases, Baltimore, USA

10.1136/sextrans-2019-sti.661

Background Observational studies have demonstrated a dosedependent association between vaginal douching and bacterial vaginosis. We sought to estimate the effect of douching cessation on the vaginal microbiota in a pilot crossover study.

Methods Thirty-two women self-collected vaginal swabs twiceweekly ( $n=950)$ during a douching observational phase ("D", 4 weeks), followed by douching cessation (“DC”, 12 weeks). Vaginal microbiota were characterized by $16 \mathrm{~S}$ rRNA gene sequencing (V3-V4) and clustered into community state types (CSTs). A conditional logistic regression model, adjusted for menstruation and sexual behaviors, allowed each woman to serve as her own control. Wilcoxon signed-rank tests were used to evaluate paired changes in microbiota between phases. Broad-range qPCR assays provided estimates of bacterial absolute abundance per swab. A piecewise linear mixed effects model was used to assess differences in rates of change in bacterial absolute abundance before and after douching.

Results There was not a statistically significant change in the odds of Lactobacillus-dominated CSTs comparing DC to D (aOR 0.54, 95\% CI: 0.27-1.11). There were no significant changes for four individual Lactobacillus spp. and no meaningful changes in other taxa investigated. The rates of change in bacterial absolute abundance was not significantly different in samples collected 3 days before and after douching $(\mathrm{p}=0.46)$. Women who had a Lactobacillus-dominated CST at baseline experienced shifts to low-Lactobacillus CST in DC, and vice versa for women who had a low-Lactobacillus CST at baseline (interaction on entry CST, p-value $<0.02$ ), however, these findings were driven by changes occurring in the final weeks.

Conclusion In this pilot study, douching cessation was not associated with major changes in vaginal microbiota. Shifts in Lactobacillus-dominance may represent regression to the mean as the shifts occurred late in DC, giving ample time for fluctuations. Disparate findings between this study and prior analyses using Nugent score may be related to low-Lactobacillus CSTs receiving low/intermediate Nugent scores.

Disclosure No significant relationships.

\section{P591 THE EFFECT OF HORMONAL CONTRACEPTION ON THE VAGINAL MICROBIOTA OVER 2 YEARS}

${ }^{1}$ Susan Tuddenham, ${ }^{1}$ Khalil Ghanem, ${ }^{2}$ Pawel Gajer, ${ }^{2}$ Courtney Robinson, ${ }^{2}$ Jacques Ravel, ${ }^{2}$ Rebecca Brotman. 'Johns Hopkins, Infectious Diseases, Baltimore, USA; ${ }^{2}$ University of Maryland, Institute of Genome Sciences, Baltimore, USA

\subsection{6/sextrans-2019-sti.662}

Background Despite widespread use, the effect of hormonal contraception $(\mathrm{HC})$ on the vaginal microbiota (VMB) is understudied. We compared VMB in a longitudinal observational study of women during intervals on and off HC.

Methods Women stopping and starting any form of $\mathrm{HC}$ and women off HC (controls) collected vaginal swabs twice-weekly for 2 weeks prior to 7 study visits over 2 years. 16S rRNA gene sequencing was conducted, and the VMB was categorized into 7 community state types (CSTs): 4 dominated by Lactobacillus spp, and 3 by Streptococcus spp (CST VI), Bifidobacterium spp (CST VII), or a variety of anaerobes (CST IV). Mixed effects logistic regression models assessed differences in CST proportions. Bayesian double exponential random effects models estimated differences between stability indices within $\mathrm{HC}$ and control subjects (measured by median Jensen-Shannon distance [MJSD] from the subject's own centroid and from the centroid of CST I [L. crispatus-dominated]).

Results 4185 samples from 105 women (73 HC, 32 controls) were available for analysis. The VMB was more stable in women on $\mathrm{HC}$ as compared to controls (MJSD 0.16 vs 0.22 , $\mathrm{p}<0.01$ ) and in oral contraceptive pill users versus controls (MJSD 0.14 vs $0.22, \mathrm{p}<0.01)$. Women had increased stability after being on $\mathrm{HC}$ for $\geq 3$ months as compared to $<3$ months (MJSD difference $-0.43, \mathrm{p}<0.01$ ). Women on $\mathrm{HC}$ for $\geq 3$ months were more likely to be in CST I $(51.3 \%$ vs $37.3 \%$, $\mathrm{p}<0.01)$ and less likely to be in CST IV $(11.4 \%$ vs $22.5 \%$, $\mathrm{p}=0.01)$ than controls. Women on $\mathrm{HC} \geq 3$ months maintained 
VMB closer to CST I than controls (MJSD 0.21 vs 0.42 , $\mathrm{p}<0.01)$.

Conclusion Women on HC have more stable, Lactobacillusdominated VMB than controls. There is increased VMB stability after 3 months of HC use. Further assessment by HC type is currently being integrated into the analysis.

Disclosure No significant relationships.

\section{P592 MICROBIOTA CONCORDANCE BETWEEN MID-VAGINAL SWABS AND BOTH CLEAN- AND RANDOM-CATCH URINE SAMPLES}

${ }^{1}$ Courtney Robinson*, 'Johanna Holm, ${ }^{2}$ Sarah Brown, 'Jacques Ravel, ${ }^{3}$ Khalil Ghanem, ${ }^{4}$ Rebecca Brotman. ${ }^{1}$ University of Maryland, Institute for Genome Sciences, Baltimore, USA; ${ }^{2}$ University of Maryland, Baltimore, Institute for Genome Sciences, Baltimore, USA; ${ }^{3}$ Johns Hopkins, Infectious Diseases, Baltimore, USA; ${ }^{4}$ University of Maryland, Institute of Genome Sciences, Baltimore, USA

\subsection{6/sextrans-2019-sti.663}

Background While urine has successfully been used for STI testing, it has not been routinely used in urogenital microbiota studies. This work explores whether random-catch and/or clean-catch urine could be a proxy for assessing the vaginal microbiota.

Methods In two studies, urinary and vaginal microbiota from women ages 17-45 were compared for (1) 91 participants with paired mid-vaginal swabs and random catch urine samples and (2) 99 participants with paired mid-vaginal swabs and clean catch urine samples. Microbiota composition was characterized by amplicon sequencing of the V3-V4 regions of the 16S rRNA gene. Taxonomic classification was assigned based on SILVA and SpeciateIt. Community State Types (CST) were assigned using an algorithm trained on 13,000 well-characterized samples. CST I, II, III, and V were dominated by: Lactobacillus crispatus, L. gasseri, L. iners, and L. jensenii, respectively. CST IV-A, IV-B, and IV-C represented low-Lactobacillus states. Similarity of paired urine and vaginal samples was measured at the CST-level by kappa statistics and the population-level with the Yue-Clayton theta indices.

Results We obtained 12 and 7.8 million sequences from urine and vaginal samples, respectively. At the CST-level, randomcatch and clean-catch urines were $82.4 \%$ and $81.8 \%$ concordant with paired mid-vaginal swabs, respectively. Substantial agreement was observed between urine and paired vaginal specimen $\left(\mathrm{K}_{\text {random-catch }}=0.770\right.$ and $\left.\mathrm{K}_{\text {clean-catch }}=0.743\right)$. At a population-level, average similarity of random- and clean-catch samples to paired vaginal samples indicated a high degree of similarity $(\theta=0.7496$ and 0.7565 , respectively). Comparison of the distributions of random-catch and clean-catch $\theta$ similarity scores showed no differences $(p=0.86)$.

Conclusion Bacterial compositions of random catch and clean catch urine samples showed substantial agreement to paired mid-vaginal samples assessed by CST- and community-level analyses. Random and clean catch urine samples could potentially be used as a proxy for vaginal microbiota in studies assessing the urogenital microbiota.

Disclosure No significant relationships.

\section{P593 A CROSS-SECTIONAL STUDY OF BIRTH MODE AND VAGINAL MICROBIOTA IN REPRODUCTIVE-AGE WOMEN}

${ }^{1}$ Christina Stennett, ${ }^{2}$ Typhanye Dyer, ${ }^{3}$ Xin He, ${ }^{4}$ Jacques Ravel, ${ }^{5}$ Khalil Ghanem, ${ }^{6}$ Rebecca Brotman*. 'University of Medicine School of Medicine, Institute for Genome Sciences, Baltimore, USA; ${ }^{2}$ University of Maryland, Epidemiology and Biostatistics, College Park, USA; ${ }^{3}$ University of Maryland, College Park, Epidemiology and Biostatistics, College Park, USA; ${ }^{4}$ University of Maryland School of Medicine, Institute of Genome Sciences, Baltimore, USA; ${ }^{5}$ Johns Hopkins, Infectious Diseases, Baltimore, USA; ${ }^{6}$ University of Maryland, Institute of Genome Sciences, Baltimore, USA

\subsection{6/sextrans-2019-sti.664}

Background Recent data suggests that birth mode (Cesarean section [C-section] versus vaginal delivery) is an important seeding event in the initial colonization of the human microbiome and is associated with long-term health. We sought to determine the association between $\mathrm{C}$-section delivery and vaginal microbiota in adulthood.

Methods We re-contacted 144 adult women from two concluded studies. In a phone survey, women reported their birth mode, obesity, breastfeeding, and age at menarche. Vaginal microbiota was characterized on a single baseline sample by amplicon sequencing of the V3-V4 hypervariable regions of the 16S rRNA gene and clustered into community state types (CSTs). We evaluated the association between birth mode and low versus high relative abundance of Lactobacillus spp. in logistic regression models controlling for body mass index, a significant confounder in this study.

Results 19\% $(\mathrm{n}=27)$ reported C-section delivery. Overall, Csection was non-significantly associated with increased odds of a low-Lactobacillus CST (aOR=1.22, 95\% CI: 0.45, 3.32). Because the two archived studies had different participant characteristics and inclusion criteria (interaction $p=0.048$ ), we stratified the analysis by study site. In the study with larger sample size $(n=88)$, women born via C-section had 3-fold higher odds of having low-Lactobacillus vaginal communities compared to vaginally-delivered women $(\mathrm{aOR}=3.55, \mathrm{p}=0.06$, 95\% CI: $0.97,13.02)$. No association was found in the smaller study $(\mathrm{n}=56, \mathrm{aOR}=0.19, \mathrm{p}=0.14,95 \% \quad \mathrm{CI}: 0.02$, 1.71).

Conclusion This cross-sectional study suggests a possible association between C-section and increased odds of a low-Lactobacillus vaginal microbiota in adulthood. However, this analysis is limited by relatively small sample size and lack of comparability in participant age, personal hygiene behaviors, and other characteristics between the study sites. Future longitudinal studies could better account for expected fluctuations in CST and may also explore confounders including behavioral factors and socioeconomic status known to be associated with both delivery mode and vaginal microbiota.

Disclosure No significant relationships. 